\title{
Human lung adenocarcinoma cells with an EGFR mutation are sensitive to non-autophagic cell death induced by zinc oxide and aluminium-doped zinc oxide nanoparticles
}

\author{
Kuan-Jen Bai1 ${ }^{1,2,3}$, Kai-Jen Chuang ${ }^{4,5}$, Chih-Ming Ma', Ta-Yuan Chang ${ }^{7}$ \\ and Hsiao-Chi Chuang2,3 \\ ${ }^{1}$ Division of Pulmonary Medicine, Department of Internal Medicine, Wan Fang Hospital, Taipei Medical University, \\ Taipei, Taiwan \\ ${ }^{2}$ School of Respiratory Therapy, College of Medicine, Taipei Medical University, Taipei, Taiwan \\ ${ }^{3}$ Department of Internal Medicine, School of Medicine, College of Medicine, Taipei Medical University, Taipei, Taiwan \\ ${ }^{4}$ Department of Public Health, School of Medicine, College of Medicine, Taipei Medical University, Taipei, Taiwan \\ ${ }^{5}$ School of Public Health, College of Public Health, Taipei Medical University, Taipei, Taiwan \\ ${ }^{6}$ Department of Cosmetic Application and Management, St. Mary's Junior College of Medicine, \\ Nursing and Management, Yilan County, Taiwan \\ 'Department of Occupational Safety and Health, College of Public Health, China Medical University, Taichung, Taiwan
}

(Received October 24, 2016; Accepted April 20, 2017)

\begin{abstract}
Lung cancer, mostly non-small cell lung cancer (NSCLC), is the leading cause of cancer deaths; however, efficient treatments for NSCLC remain insufficient. The objective of this study was to investigate the effects of an epidermal growth factor receptor (EGFR) mutation on autophagic cell death in human lung adenocarcinoma cells by $20-\mathrm{nm}$ zinc oxide nanoparticles (ZnONP20) and aluminumdoped $\mathrm{ZnONPs}$ (Al-ZnONP20). Two types of human lung adenocarcinoma cells were used throughout the study: wild-type EGFR A549 cells and EGFR-mutated CL1-5 cells. We observed that a significant reduction in cell viability resulting from ZnONP20 and Al-ZnONP20 occurred in A549 and CL1-5 cells after 18 and $24 \mathrm{hr}$ of exposure. A colony formation analysis showed that A549 cells re-grew after exposure to $20 \mu \mathrm{g} / \mathrm{mL}$ Al-ZnONP20. Levels of light chain 3 (LC3) II conversion were activated by ZnONP20 and AlZnONP20 in A549 cells, whereas LC3 II was inhibited by ZnONP20 and Al-ZnONP20 in CL1-5 cells. In conclusion, we have shown that human lung adenocarcinoma cells with an EGFR mutation are sensitive to ZnONP20 and Al-ZnONP20, which may have resulted in non-autophagic cell death. ZnONP20 and AlZnONP20 may have the potential for personalized therapeutics in NSCLC with an EGFR mutation.
\end{abstract}

Key words: Cell death, Epidermal growth factor receptor, Lung cancer, Nanoparticle, Zinc oxide

\section{INTRODUCTION}

Lung cancer, mostly non-small cell lung cancer (NSCLC), is the leading cause of cancer deaths; however, efficient treatments for NSCLC remain insufficient. Frontline therapies using standard platinum-based doublet combination regimens have reached a plateau, with 1 -year survival rates of only $35 \% \sim 40 \%$ and a median overall survival of 12 months recently being barely achieved (Ciuleanu et al., 2009). Although molecular targeted therapy, particularly epidermal growth factor receptor (EGFR)-tyrosine kinase inhibitors (TKIs), seem to have achieved promising results, a prolongation of overall survival compared to conventional chemotherapy as the first-line therapy for those with EGFR-mutation-positive tumors has never been demonstrated (Shepherd et al., 2005; Mok et al., 2009). This suggests that patients still need to rely on chemotherapy as part of their standard treatment. Therefore, novel therapeutic regimens need to be developed and evaluated in order to complement current treatments.

Nanotechnology has rapidly developed in importance over time, especially in biomedical applications. For example, nanomaterials were proven to exhibit

Correspondence: Hsiao-Chi Chuang (E-mail: r92841005@ntu.edu.tw) 


\section{K.-J. Bai et al.}

promising properties that allow them to improve tumor responses to chemo- and radiotherapy in cancer therapy (Beik et al., 2016; Ma and Yang, 2016). Recently, zinc oxide nanoparticles ( $\mathrm{ZnONPs}$ ) were considered to have the potential for cancer treatment (Zhang et al., 2013). Hackenberg and colleagues observed that ZnONPs followed by UVA-1 irradiation were able to induce cell death of human head and neck squamous cell carcinoma cells (Hackenberg et al., 2014). Additionally, previous reports demonstrated that ZnNOPs caused cytotoxicity in cell-specific and proliferation-dependent manners (Hanley et al., 2008; Premanathan et al., 2011; Ostrovsky et al., 2009). Therefore, it is important to investigate the efficiency of treating different types of cancer cells with ZnONPs.

Previous findings suggested that autophagy may play a protective role in the early stage of cancer (Sui et al., 2013; Yang et al., 2011). Autophagy is also reported to induce lethal mechanisms in tumor cells (Morselli et al., 2009). Chemotherapy- and metabolic stress-induced activation of autophagy may result in the survival of tumor cells, leading to resistance in cancer cells. On the contrary, autophagy inhibition may represent a major therapeutic target for chemosensitization (Yang et al., 2011). Therefore, oncogenesis and tumor survival are influenced by the molecular machinery that controls autophagy.

Genetic variations leading to overexpression or mutations of the EGFR play essential roles in the occurrence and progression of lung cancers such as NSCLC (Li et al., 2016a). EGFR-TKIs are recognized as a standard NSCLC therapy and have great clinical benefits (Farhat and Houhou, 2013). However, primary or acquired drug resistance remains a big challenge to the success of TKI therapy in NSCLC. Therefore, ZnONPs for NSCLC have a great potential due to their unique characteristics. The objectives of this study were: (1) to investigate the effects of ZnONPs and aluminum-doped ZnONPs (Al-ZnONPs) on the cell viability and proliferation in wild-type EGFR A549 cells and EGFR-mutated CL1-5 cells; and (2) to observe the expression of microtubule-associated protein 1A/1B-light chain 3 (LC3) in A549 and CL1-5 cells after $\mathrm{ZnONP}$ and $\mathrm{Al}-\mathrm{ZnONP}$ exposure.

\section{MATERIALS AND METHODS}

\section{Particles and reagent sources}

The physical properties of ZnONP20 and Al-ZnONP20, obtained from Nanostructured \& Amorphous Materials (Houston, TX, USA), used in this study were previously reported (Pan et al., 2014). All of the other reagents were obtained from Sigma (St. Louis, MO, USA), unless the source is explicitly stated.

\section{Particle characterization}

Inspect $^{\text {TM }}$ FE-SEM (JEOL 2100, Jeol, Tokyo, Japan) was used to characterize the morphology of the particles. Preparation of the FE- scanning electron microscopic (SEM) samples was previously described (Chuang et al., 2011). Briefly, nanoparticles adhered to $12-\mathrm{mm}$ carbon sticky tabs, which were fixed on 13-mm aluminum SEM stubs. Nanoparticles were coated with platinum $(\mathrm{Pt})$ to an average thickness of $10 \mathrm{~nm}$ using a sputter-coater and then imaged. FE-SEM was operated at an accelerating voltage of $15 \mathrm{kV}$ and a 2.5-nm spot size. An elemental analysis was conducted using the EDX Genesis Microanalysis System. Specific surface areas of nanoparticles were calculated by the Brunauer-Emmett-Teller (BET) method at a relative pressure $(\mathrm{P} / \mathrm{P} 0)$ of 0.99 using a specific surface area and pore size distribution analyzer (ASAP 2020, Micromeritics Instrument, Norcross, GA, USA).

\section{Cell culture and treatment}

Human lung adenocarcinoma A549 cells (with the wild-type EGFR) were obtained from the American Type Culture Collection (ATCC, Manassas, VA, USA). Human lung adenocarcinoma CL1-5 cells (with an EGFR mutation) were originally established from a single cell clone isolated from lung adenocarcinoma tissues of a patient with a poorly differentiated adenocarcinoma (Lee-Huang et al., 2000), which was kindly provided by Dr. C-T. Yeh (Shuang Ho Hospital, New Taipei City, Taiwan). A549 and CL1-5 cells are commonly used for EGFR studies on lung cancer. (Lee et al., 2011) These cells were seeded in surface-treated, 96-well transwells at a density of $10^{5}$ cells $/ \mathrm{mL}$ and incubated for $24 \mathrm{hr}$ (BD Biosciences, Oxford, UK). Cells were cultured in RPMI medium containing 10\% fetal bovine serum (FBS), penicillin, and streptomycin and were incubated at $37^{\circ} \mathrm{C}$, 95\% humidity, and 5\% $\mathrm{CO}_{2}$. ZnONP20 and Al-ZnONP20 solutions were freshly prepared in $10 \%$ FBS-containing cell medium. To determine the cell viability, cells were incubated with $0,20,50,150$, and $300 \mu \mathrm{g} / \mathrm{mL} \mathrm{ZnONP} 20$ or Al-ZnONP20 at $37^{\circ} \mathrm{C}$ for $1,6,18$, and $24 \mathrm{hr}$ in a humidified atmosphere with $5 \% \mathrm{CO}_{2}$. For the colony formation assay, cells were incubated with $0,20,50$, and $150 \mu \mathrm{g} / \mathrm{mL} \mathrm{ZnONP} 20$ or Al-ZnONP20. For the Western blot analysis, cells were incubated with $0,20,50$, and $150 \mu \mathrm{g} / \mathrm{mL}$ ZnONP20 or Al-ZnONP20. Each experiment was conducted at least in triplicate.

\section{Cell viability}

A sulforhodamine B (SRB) colorimetric assay was used 
Inhibition of cell survival by ZnONPs and Al-ZnONPs in cells with an EGFR mutation

to investigate cell viability according a previous method (Vichai and Kirtikara, 2006). Briefly, cells were fixed with $10 \%$ (wt/vol) trichloroacetic acid and stained for $30 \mathrm{~min}$. The protein-bound dye was dissolved in $10 \mathrm{mM}$ of a Tris base solution after removing excess dye. The OD was measured at $510 \mathrm{~nm}$ using a microplate reader (Bio-Rad Model 680 microplate reader, Hertfordshire, UK). Cell viability is presented as a percentage (\%) after adjusting for the control.

\section{Colony formation assay}

The protocol of the colony formation assay was described previously (Franken et al., 2006). Briefly, 500 cells were resuspended in RPMI medium and seeded in six-well surface-coated plates following a 24-hr incubation. Cells were exposed to 0, 20, 50, and $150 \mu \mathrm{g} / \mathrm{mL}$ of particles for 5 days. Next, cells were washed and cultured for a further 9 days. Finally, cells were washed and fixed with $3.7 \%$ paraformaldehyde following staining with $0.05 \%$ crystal violet. Plates were stained with $0.5 \mathrm{mg} / \mathrm{mL}$ p-iodonitrotetrazolium violet. Colonies with a diameter of $>1 \mathrm{~mm}$ were counted. Triplicate samples were used in the experiment. Images were then analyzed for the colony formation rate (\%) using ImageJ software (vers. 1.49, Bethesda, MD, USA) (Fertsch-Gapp et al., 2011).

\section{Western blot analysis}

Western blot analyses were conducted as described previously (Chen et al., 2004). Briefly, cells were cultured in 6-cm dishes for nanoparticle exposure. Proteins isolated from lysed cells were subjected to sodium dodecylsulfate polyacrylamide gel electrophoresis (SDS-PAGE) and were electrotransferred onto polyvinylidene difluoride (PVDF) membranes (Millipore, Darmstadt, Germany). Primary antibodies for LC3 $(1: 1000)$ and $\beta$-catenin (1:1000) were obtained from Cell Signaling (Danvers, MA, USA). Antirabbit (1:2000) horseradish peroxidase (HRP)-conjugated secondary antibodies were obtained from Chemicon International (Billerica, MA, USA). Blots were then blocked with 5\% skim milk in PBST and were probed with primary antibodies overnight at $4^{\circ} \mathrm{C}$. An HRPlabeled secondary antibody was then incubated for $60 \mathrm{~min}$ at room temperature and washed with TBST. Enhanced chemiluminescence (ECL) Western blotting reagents were used, followed by imaging using the BioSpectrum Imaging System (UVP, Upland, CA, USA). Quantitative data were obtained using ImageJ software (Fertsch-Gapp et al., 2011).

\section{Statistical analysis}

Data are expressed as the mean \pm standard deviation (S.D.). A one-way analysis of variance (ANOVA) with Tukey's post-hoc test was used for comparisons among multiple values. Statistical analyses were performed using GraphPad vers. 5 for Windows. The level of significance was set to $p<0.05$.

\section{RESULTS}

\section{Characterization of ZnONP20 and AI-ZnONP20}

ZnONP20 (with an average diameter of $20 \mathrm{~nm}$ ) and Al-ZnONP20 (with an average diameter of 20 40 nm) were characterised using FE-SEM coupled with EDX (Fig. 1). The purity was $\mathrm{ZnO}=99.5 \mathrm{wt} \%$ for $\mathrm{ZnONP} 20$ and $\mathrm{ZnO}: \mathrm{Al}_{2} \mathrm{O}_{3}=98: 2 \mathrm{wt} \%$ for Al-ZnONP20, which are values provided by the supplier. ZnONP20 presented a hexagonal structure, whereas an irregular morphology of Al-ZnONP20 was observed. Both ZnONP20 and Al$\mathrm{ZnONP} 20$ were $\mathrm{Zn}$ dominant, but Al-ZnONP20 contained a minor amount of Al. Surface areas of ZnONP20 and Al$\mathrm{ZnONP} 20$ were 22.3 and $3.6 \mathrm{~m}^{2} / \mathrm{g}$, respectively.

\section{Cell viability and proliferation}

To investigate the particle toxicity caused by ZnONP20 and Al-ZnONP20 in A549 and CL1-5 cells, cell viability and colony formation were determined. First, we observed that a significant reduction in cell viability resulted from $\mathrm{ZnONP} 20$ at $300 \mu \mathrm{g} / \mathrm{mL}$ for 18 and $24 \mathrm{hr}$ of exposure in A549 cells $(p<0.05)$, whereas cell viability of CL1-5 cells significantly decreased with 20, 50, 150, and $300 \mu \mathrm{g} / \mathrm{mL}$ after 18 and $24 \mathrm{hr}$ of exposure $(p<0.05)$ (Fig. 2). The cell viability of A549 cells decreased with Al-ZnONP20 at 150 and $300 \mu \mathrm{g} / \mathrm{mL}$ for 18 and $24 \mathrm{hr}$ of exposure $(p<0.05)$, whereas Al-ZnONP20 significantly reduced the cell viability of CL-15 cells when exposed to 150 and $300 \mu \mathrm{g} / \mathrm{mL}$ for $6 \mathrm{hr}$ and $20,50,150$ and $300 \mu \mathrm{g} / \mathrm{mL}$ for 18 and $24 \mathrm{hr}(p<0.05)$. The results suggest that A549 cells were more resistant to ZnONP20 than were CL1-5 cells, and Al-ZnONP20 caused higher cell death than ZnONP20.

Next, the effects on cell proliferation by ZnONP20 and Al-ZnONP20 in A549 and CL1-5 cells were determined using a colony formation assay. As shown in Fig. 3, ZnONP20 significantly affected the growth rates of A549 and CL1-5 cells at 20,50, and $150 \mu \mathrm{g} / \mathrm{mL}$. Notably, the growth rate of A1-ZnONP20-treated A549 cells was faster at $20 \mu \mathrm{g} / \mathrm{mL}$ than that of CL1-5 cells. The colony formation rate of A549 cells was inhibited by ZnONP20, but not by Al-ZnONP20 at $20 \mu \mathrm{g} / \mathrm{mL}$. The colony formation rate of CL1-5 cells was inhibited by both ZnONP20 and 


\section{K.-J. Bai et al.}
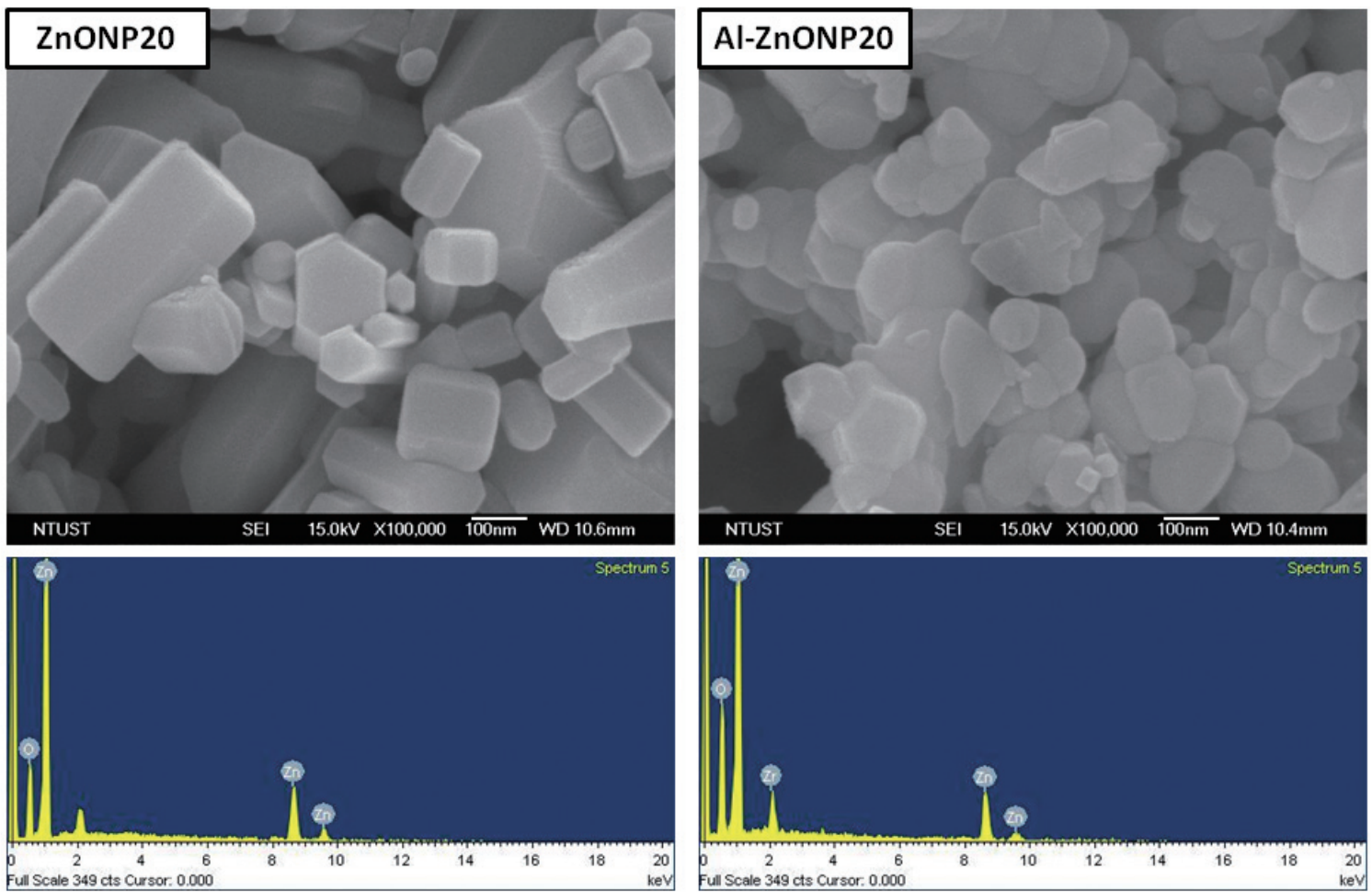

Fig. 1. FE-SEM and EDX analyses of 20-nm zinc oxide nanoparticles (ZnONP20) and aluminum-doped (Al)-ZnONP20. ZnONP20 presented a hexagonal structure, whereas an irregular morphology of Al-ZnONP20 was observed. Both nanoparticles were Zn dominant.

\section{Al-ZnONP20}

\section{LC3 expression}

Expressions of LC3 II (after controlling for LC3 I) after exposure to ZnONP20 and Al-ZnONP20 in A549 and CL1-5 cells were determined. As shown in Fig. 4, expression of LC3 II increased after exposure to $\mathrm{ZnONP20}$ in A549 cells, particularly at $50 \mu \mathrm{g} / \mathrm{mL}$ $(p<0.05)$, whereas LC3 II was steadily activated by Al-ZnONP20 and reached a significant increase at $150 \mu \mathrm{g} / \mathrm{mL}(p<0.05)$. Although the expression of LC3 II slightly increased by exposure to ZnONP20 and AlZnONP20 in CL1-5 cells, the increase in LC3 II was not significant. LC3 conversion caused by ZnONP20 and AlZnONP20 was found in A549 cells, but not observed in CL1-5 cells, suggesting that distinct effects of LC3 activation by exposure to $\mathrm{ZnONP20}$ and Al-ZnONP20 occurred in A549 and CL1-5 cells.

\section{DISCUSSION}

Autophagy is considered to be an important pathway in lung disease pathogenesis and therapeutics. ZnONPs are considered to have potential for cancer therapy. In the present study, we observed that ZnONP20 and AlZnONP20 may have potential for treating lung adenocarcinoma cells with an EGFR mutation. Three major findings are reported in the present study: (1) the cell viability and proliferation of CL1-5 cells with an EGFR mutation were sensitive to ZnONP20 and Al-ZnONP20; (2) cell viability of A549 cells with the wild-type EGFR was resistant to ZnONP20 and Al-ZnONP20; and (3) autophagy was activated by ZnONP20 and Al-ZnONP20 in A549 cells with the wild-type EGFR, whereas autophagy was inhibited by ZnONP20 and Al-ZnONP20 in CL1-5 cells with an EGFR mutation.

To investigate the effects of ZnONPs on autophagic cell death in lung adenocarcinoma cells with or without an EGFR mutation, the 20-nm-diameter engineered ZnONPs (ZnONP20) and Al-ZnONP (Al-ZnONP20) were used throughout the study. These nanoparticles present differences in morphology as observed in this study. Their physicochemical properties, including size, elements, electronic properties, zinc concentration, and $\mathrm{pH}$, were 
Inhibition of cell survival by ZnONPs and Al-ZnONPs in cells with an EGFR mutation
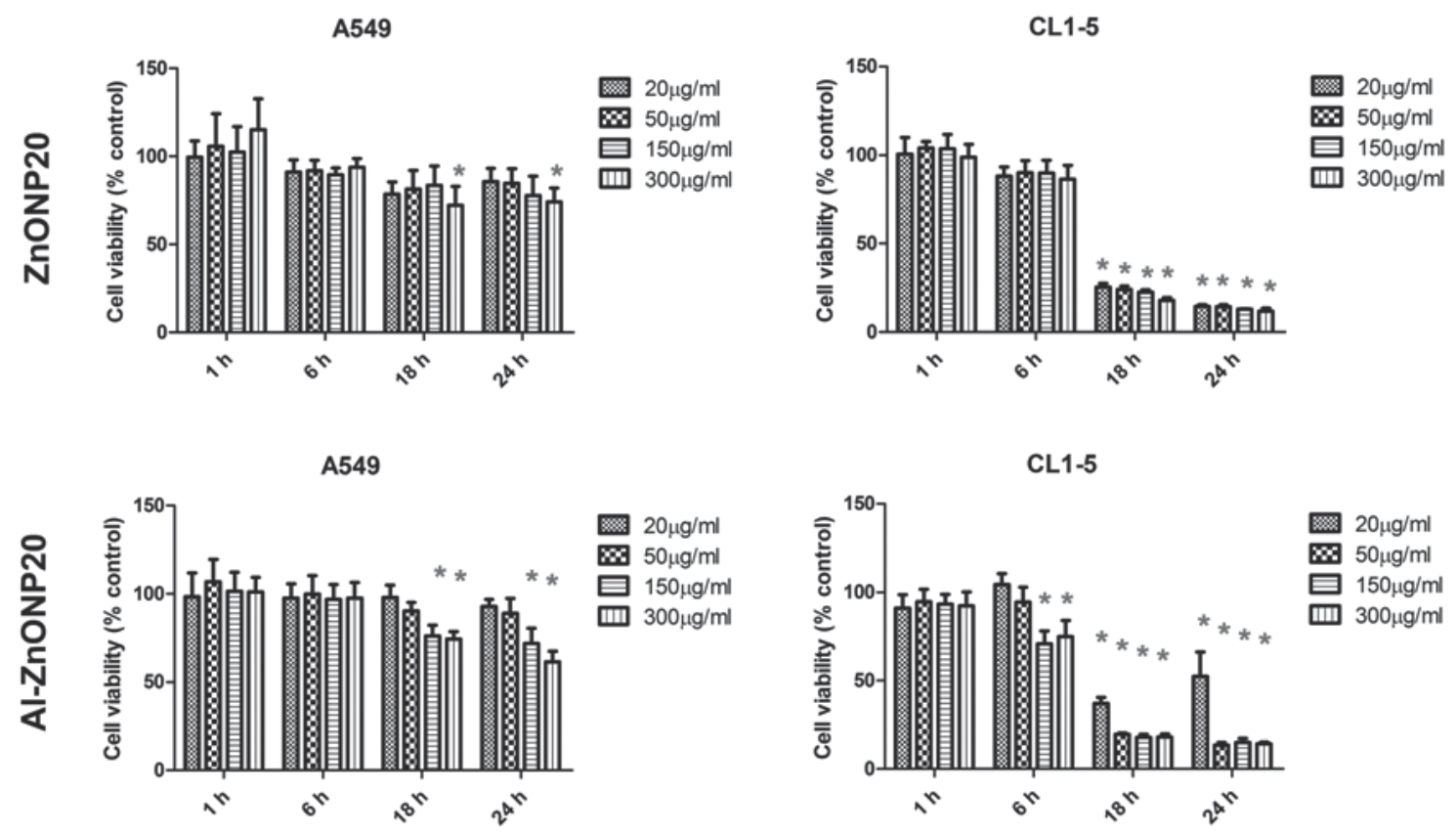

Fig. 2. Effects of 20-nm zinc oxide nanoparticles (ZnONP20) and aluminum-doped (Al)-ZnONP20 on cell viability of A549 (with the wild-type epidermal growth factor receptor (EGFR)) and CL1-5 (with an EGFR mutation) cells. Cell viability was determined in response to ZnONP20 and Al-ZnONP20 at 0,20,50, 150, and $300 \mu \mathrm{g} / \mathrm{mL}$ for 1, 6, 18, and $24 \mathrm{hr}$. A549 cells were more resistant to ZnONP20 than were CL1-5 cells, and Al-ZnONP20 caused higher cell death than ZnONP20. $* p<0.05$.
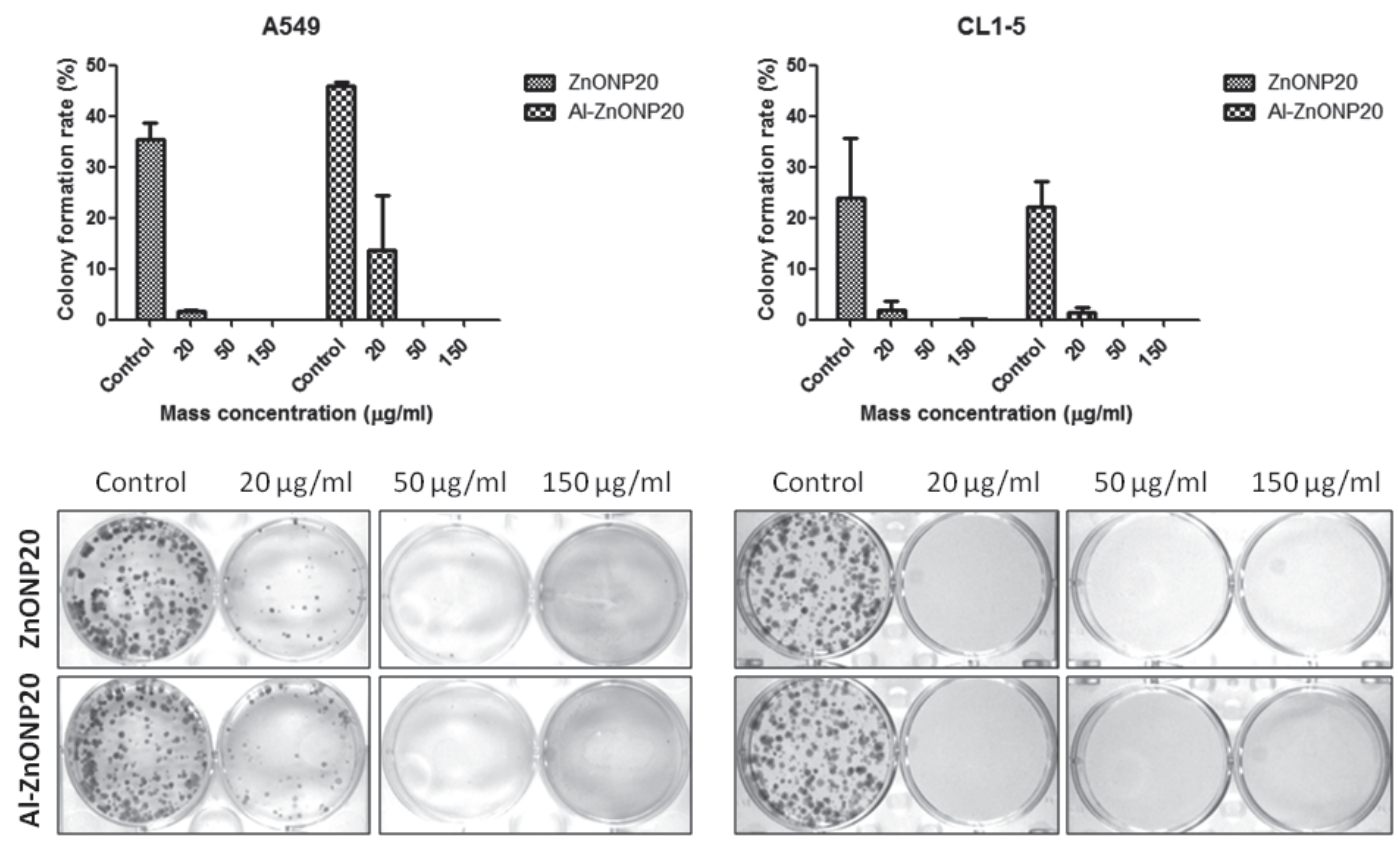

Fig. 3. Effects of 20-nm zinc oxide nanoparticles (ZnONP20) and aluminum-doped (Al)-ZnONP20 on cell proliferation of A549 (with the wild-type epidermal growth factor receptor (EGFR)) and CL1-5 (with an EGFR mutation) cells. Colony formation was analyzed in response to ZnONP20 and Al-ZnONP20 at $20 \mu \mathrm{g} / \mathrm{mL}$. The colony formation rate of CL1-5 cells was significantly inhibited by both ZnONP20 and Al-ZnONP20. 
A549

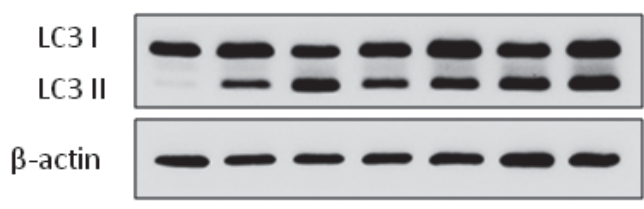

$\mathrm{ZnONP20}(\mu \mathrm{g} / \mathrm{ml})-2050150-\ldots$
CL1-5

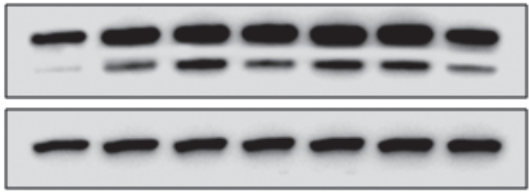

$-2050150--$

$-\quad-\quad-2050150$

$\mathrm{Al}-\mathrm{ZnONP20}(\mu \mathrm{g} / \mathrm{ml})-\quad-\quad-\quad 20 \quad 50150$
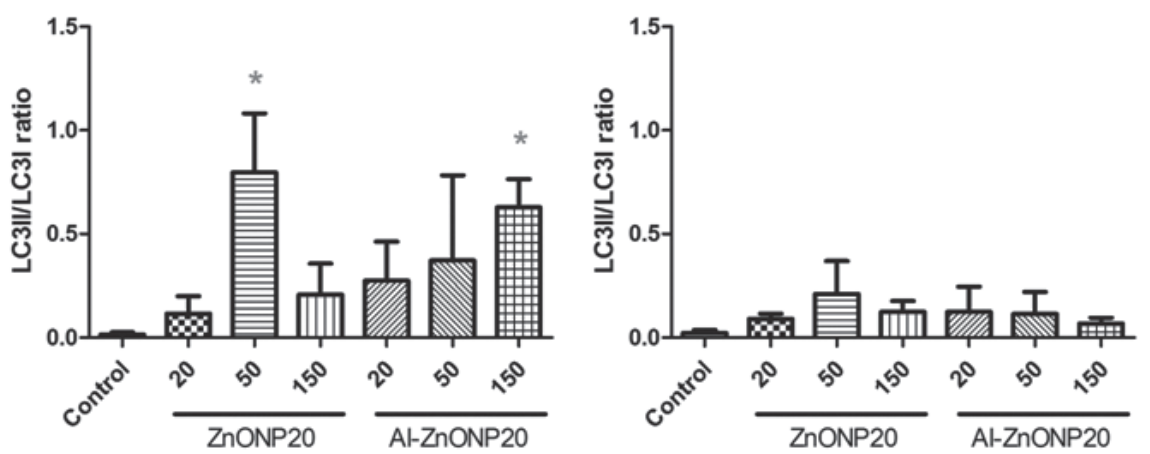

Fig. 4. Effects of 20-nm zinc oxide nanoparticles (ZnONP20) and aluminum-doped (Al)-ZnONP20 on light chain 3 (LC3) expression of A549 (with the wild-type epidermal growth factor receptor (EGFR)) and CL1-5 (with an EGFR mutation) cells. LC3 II expression (adjusted by LC3 I) was determined after exposure of A549 and CL1-5 cells to ZnONP20 and Al-ZnONP20 at $0,20,50$, and $150 \mu \mathrm{g} / \mathrm{mL}$ for $24 \mathrm{hr}$. LC3 conversion caused by ZnONP20 and Al-ZnONP20 significantly increased in A549 cells, but was inhibited in CL1-5 cells. ${ }^{*} p<0.05$.

reported previously (Pan et al., 2014). To reduce particle aggregation/agglomeration when suspended in solution for toxicity testing, serum-containing cell medium was used throughout the study. Serum proteins act as an excellent dispersant for nanoparticles in biological solutions (Cho et al., 2012; Chuang et al., 2014), but the dispersive ability was only maintained for a short time, after which aggregation/agglomeration recurred (Pan et al., 2014). Physicochemical characteristics of nanoparticles play important roles in regulating nanoparticle toxicity (Buerki-Thurnherr et al., 2013; Pan et al., 2014). Solubility, for example, is recognized as an important determinant of regulating $\mathrm{ZnONP}$ toxicity. In the present study, Al-ZnONP20 was used to slow $\mathrm{Zn}$ ion leaching during exposure to cells, which was served as control nanoparticles. Notably, the long-term difference of nanotoxicity caused by their physicochemistry between ZnONP20 and Al-ZnONP20 might not be significant due to plateau levels of $\mathrm{Zn}$ ion in biological systems. Therefore, the solubility of the Zn-based may influence the bioreactivity in vitro, which should be considered in future works.

The ability to avoid cell death by cancer cells is a hallmark of cancer, leading to cancer development and progression. Therefore, inhibition of cell viability and re-growth is an essential indicator for the treatment efficiency in lung cancer. Lung cancer is a malignant tumor, characterized by uncontrolled cell growth in lung tissues (CDC, 2015). Clinically, mutations in the EGFR were observed in $15 \%$ 20\% of lung adenocarcinomas (Siegelin and Borczuk, 2014). In the present study, A549 cells with a wild-type EGFR and CL1-5 cells with an EGFR mutation were exposed to ZnONP20 and Al-ZnONP20. We observed that both ZnONP20 and Al-ZnNOP20 were efficient at reducing cell viability and inhibiting cell regrowth in CL1-5 cells. Also, ZnONP20 and Al-ZnNOP20 were able to reduce cell viability, but A549 cells exhibited re-growth after exposure to Al-ZnONP20. Consistent with previous reports, ZnONPs are able to decrease cell viability (Kumar et al., 2015; Wang et al., 2014; Meyer et al., 2011), suggesting the potential for cancer therapy. Additionally, Hackenberg and colleagues found that ZnNOPs were able to induce the photocatalytic death of head and neck squamous cell carcinoma cells (Hackenberg et al., 2014). Therefore, based on our find- 
Inhibition of cell survival by ZnONPs and Al-ZnONPs in cells with an EGFR mutation

ings, both ZnONP20 and Al-ZnONP20 may have the potential for treating NSCLC with an EGFR mutation.

Regulation of autophagy is associated with the survival of tumor cells and therapeutic efficiency. Recently, autophagic cell death was reported to be associated with ZnONP exposure (Hackenberg et al., 2014; Johnson et al., 2015; Yu et al., 2013). However, in the present study, activation of autophagy caused by ZnNOP20 and Al-ZnNOP20 was only observed in A549 cells, but not in CL1-5 cells. LC3 was indicated to increase in expression of autophagy after exposure to metal-based nanoparticles, including $\mathrm{As}, \mathrm{Cd}, \mathrm{Cr}, \mathrm{Hg}$, and $\mathrm{Fe}$ (Chatterjee et al., 2014). Together, we suggest that cellspecific autophagy was associated with exposure to ZnONP20 and Al-ZnONP20. Previous studies showed activation of LC3 expression in CL1-5 cells for drug screening (Li et al., 2016b; Chen et al., 2015), suggesting that autophagy functions well in CL1-5 cells. Based on our observations, we hypothesized that autophagy inhibition occurred due to ZnNOP20 and Al-ZnONP20 in CL1-5 cells. The difference between cell death in A549 and CL1-5 cells may have resulted from the EGFR mutation status. In other words, the EGFR mutation in CL1-5 cells makes them sensitive to ZnONP20 and Al$\mathrm{ZnONP} 20$ by reducing cell viability and inhibiting cell regrowth via a non-autophagic pathway. The results imply that $\mathrm{ZnONP20}$ and Al-ZnONP20 may have the potential to treat NSCLC with an EGFR mutation. However, confirmation experiments are required to investigate the upand downstream pathways in autophagy.

In conclusion, we showed that human lung adenocarcinoma cells with an EGFR mutation are sensitive to ZnONP20 and Al-ZnONP20, which may have resulted in non-autophagic cell death. On the other hand, lung adenocarcinoma cells with the wild-type EGFR were resistant to ZnONP20 and Al-ZnONP20, and may have been protected by an autophagy pathway. To investigate the role of autophagy in response to ZnONP20 and Al-ZnONP20, in vivo experiments should be conducted in further works. Our results demonstrate that ZnONP20 and Al-ZnONP20 may have the potential for personalized therapeutics in NSCLC with an EGFR mutation based on the safety dose in human. Further research on other types of lung cancer cells and in vivo experiments are required.

\section{ACKNOWLEDGMENTS}

The authors wish to thank Miss Yi-Syuan Lin for technical assistance with this research. Also, the authors wish to thank Dr. Chia-Hung Hou for the BET analysis. This study was funded by the Ministry of Science and
Technology of Taiwan (MOST103-2314-B-038-018 and MOST104-2621-M-038-002-MY3).

Conflict of interest---- The authors declare that there is no conflict of interest.

\section{REFERENCES}

Beik, J., Abed, Z., Ghoreishi, F.S., Hosseini-Nami, S., Mehrzadi, S., Shakeri-Zadeh, A. and Kamrava, S.K. (2016): Nanotechnology in hyperthermia cancer therapy: From fundamental principles to advanced applications. J. Control. Release, 235, 205-221.

Buerki-Thurnherr, T., Xiao, L., Diener, L., Arslan, O., Hirsch, C., Maeder-Althaus, X., Grieder, K., Wampfler, B., Mathur, S., Wick, P. and Krug, H.F. (2013): In vitro mechanistic study towards a better understanding of $\mathrm{ZnO}$ nanoparticle toxicity. Nanotoxicology, 7, 402-416.

CDC (2015): Lung Cancer.

Chatterjee, S., Sarkar, S. and Bhattacharya, S. (2014): Toxic metals and autophagy. Chem. Res. Toxicol., 27, 1887-1900.

Chen, B.C., Chang, Y.S., Kang, J.C., Hsu, M.J., Sheu, J.R., Chen, T.L., Teng, C.M. and Lin, C.H. (2004): Peptidoglycan induces nuclear factor-kappaB activation and cyclooxygenase-2 expression via Ras, Raf-1, and ERK in RAW 264.7 macrophages. J. Biol. Chem., 279, 20889-20897.

Chen, Y.K., Wang, H.C., Ho, C.T., Chen, H.Y., Li, S., Chan, H.L., Chung, T.W., Tan, K.T., Li, Y.R. and Lin, C.C. (2015): 5-demethylnobiletin promotes the formation of polymerized tubulin, leads to $\mathrm{G} 2 / \mathrm{M}$ phase arrest and induces autophagy via JNK activation in human lung cancer cells. J. Nutr. Biochem., 26, 484-504.

Cho, W.S., Duffin, R., Poland, C.A., Duschl, A., Oostingh, G.J., Macnee, W., Bradley, M., Megson, I.L. and Donaldson, K. (2012): Differential pro-inflammatory effects of metal oxide nanoparticles and their soluble ions in vitro and in vivo; zinc and copper nanoparticles, but not their ions, recruit eosinophils to the lungs. Nanotoxicology, 6, 22-35.

Chuang, H.C., Jones, T.P., Lung, S.C. and BéruBé, K.A. (2011): Soot-driven reactive oxygen species formation from incense burning. Sci. Total Environ., 409, 4781-4787.

Chuang, H.C., Juan, H.T., Chang, C.N., Yan, Y.H., Yuan, T.H., Wang, J.S., Chen, H.C., Hwang, Y.H., Lee, C.H. and Cheng, T.J. (2014): Cardiopulmonary toxicity of pulmonary exposure to occupationally relevant zinc oxide nanoparticles. Nanotoxicology, 8, 593-604.

Ciuleanu, T., Brodowicz, T., Zielinski, C., Kim, J.H., Krzakowski, M., Laack, E., Wu, Y.L., Bover, I., Begbie, S., Tzekova, V., Cucevic, B., Pereira, J.R., Yang, S.H., Madhavan, J., Sugarman, K.P., Peterson, P., John, W.J., Krejcy, K. and Belani, C.P. (2009): Maintenance pemetrexed plus best supportive care versus placebo plus best supportive care for non-small-cell lung cancer: a randomised, double-blind, phase 3 study. Lancet, 374, 14321440 .

Farhat, F.S. and Houhou, W. (2013): Targeted therapies in non-small cell lung carcinoma: what have we achieved so far? Ther. Adv. Med. Oncol., 5, 249-270.

Fertsch-Gapp, S., Semmler-Behnke, M., Wenk, A. and Kreyling, W.G. (2011): Binding of polystyrene and carbon black nanoparticles to blood serum proteins. Inhal. Toxicol., 23, 468-475.

Franken, N.A., Rodermond, H.M., Stap, J., Haveman, J. and van Bree, C. (2006): Clonogenic assay of cells in vitro. Nature 


\section{K.-J. Bai et al.}

Protoc., 1, 2315-2319.

Hackenberg, S., Scherzed, A., Gohla, A., Technau, A., Froelich, K., Ginzkey, C., Koehler, C., Burghartz, M., Hagen, R. and Kleinsasser, N. (2014): Nanoparticle-induced photocatalytic head and neck squamous cell carcinoma cell death is associated with autophagy. Nanomedicine, 9, 21-33.

Hanley, C., Layne, J., Punnoose, A., Reddy, K.M., Coombs, I., Coombs, A., Feris, K. and Wingett, D. (2008): Preferential killing of cancer cells and activated human $\mathrm{T}$ cells using $\mathrm{ZnO}$ nanoparticles. Nanotechnology, 19, 295103.

Johnson, B.M., Fraietta, J.A., Gracias, D.T., Hope, J.L., Stairiker, C.J., Patel, P.R., Mueller, Y.M., McHugh, M.D., Jablonowski, L.J., Wheatley, M.A. and Katsikis, P.D. (2015): Acute exposure to $\mathrm{ZnO}$ nanoparticles induces autophagic immune cell death. Nanotoxicology, 9, 737-748.

Kumar, A., Najafzadeh, M., Jacob, B.K., Dhawan, A. and Anderson, D. (2015): Zinc oxide nanoparticles affect the expression of p53, Ras p21 and JNKs: an ex vivo/in vitro exposure study in respiratory disease patients. Mutagenesis, 30, 237-245.

Lee-Huang, S., Huang, P.L., Sun, Y., Chen, H.C., Kung, H.F., Huang, P.L. and Murphy, W.J. (2000): Inhibition of MDA-MB231 human breast tumor xenografts and HER2 expression by anti-tumor agents GAP31 and MAP30. Anticancer Res., 20, 653-659.

Lee, J.Y., Lee, Y.M., Chang, G.C., Yu, S.L., Hsieh, W.Y., Chen, J.J., Chen, H.W. and Yang, P.C. (2011): Curcumin induces EGFR degradation in lung adenocarcinoma and modulates p38 activation in intestine: the versatile adjuvant for gefitinib therapy. PLoS One, 6, e23756.

Li, Y., Yang, Z., Li, W., Xu, S., Wang, T., Wang, T., Niu, M., Zhang, S., Jia, L. and Li, S. (2016a): TOPK promotes lung cancer resistance to EGFR tyrosine kinase inhibitors by phosphorylating and activating c-Jun. Oncotarget, 7, 6748-6764.

Li, Y.R., Li, S., Ho, C.T., Chang, Y.H., Tan, K.T., Chung, T.W., Wang, B.Y., Chen, Y.K. and Lin, C.C. (2016b): Tangeretin derivative, 5-acetyloxy-6,7,8,4'-tetramethoxyflavone induces G2/M arrest, apoptosis and autophagy in human non-small cell lung cancer cells in vitro and in vivo. Cancer Biol.Ther., 17, 48-64.

Ma, D.D. and Yang, W.X. (2016): Engineered nanoparticles induce cell apoptosis: potential for cancer therapy. Oncotarget, 7, 40882-40903.

Meyer, K., Rajanahalli, P., Ahamed, M., Rowe, J.J. and Hong, Y. (2011): ZnO nanoparticles induce apoptosis in human dermal fibroblasts via p53 and p38 pathways. Toxicol. In Vitro, 25, 1721-1726.

Mok, T.S., Wu, Y.L., Thongprasert, S., Yang, C.H., Chu, D.T., Saijo, N., Sunpaweravong, P., Han, B., Margono, B., Ichinose, Y., Nishiwaki, Y., Ohe, Y., Yang, J.J., Chewaskulyong, B., Jiang, H., Duffield, E.L., Watkins, C.L., Armour, A.A. and Fukuoka,
M. (2009): Gefitinib or carboplatin-paclitaxel in pulmonary adenocarcinoma. N. Eng. J. Med., 361, 947-957.

Morselli, E., Galluzzi, L., Kepp, O., Vicencio, J.M., Criollo, A., Maiuri, M.C. and Kroemer, G. (2009): Anti- and pro-tumor functions of autophagy. Biochim. Biophys. Acta, 1793, 1524-1532.

Ostrovsky, S., Kazimirsky, G., Gedanken, A. and Brodie, C. (2009): Selective cytotoxic effect of $\mathrm{ZnO}$ nanoparticles on glioma cells. Nano Research, 2, 882-890.

Pan, C.H., Liu, W.T., Bien, M.Y., Lin, I.C., Hsiao, T.C., Ma, C.M., Lai, C.H., Chen, M.C., Chuang, K.J. and Chuang, H.C. (2014): Effects of size and surface of zinc oxide and aluminum-doped zinc oxide nanoparticles on cell viability inferred by proteomic analyses. Int. J. Nanomedicine, 9, 3631-3643.

Premanathan, M., Karthikeyan, K., Jeyasubramanian, K. and Manivannan, G. (2011): Selective toxicity of $\mathrm{ZnO}$ nanoparticles toward Gram-positive bacteria and cancer cells by apoptosis through lipid peroxidation. Nanomedicine, 7, 184-192.

Shepherd, F.A., Rodrigues Pereira, J., Ciuleanu, T., Tan, E.H., Hirsh, V., Thongprasert, S., Campos, D., Maoleekoonpiroj, S., Smylie, M., Martins, R., van Kooten, M., Dediu, M., Findlay, B., Tu, D., Johnston, D., Bezjak, A., Clark, G., Santabárbara, P., Seymour, L. and National Cancer Institute of Canada Clinical Trials Group. (2005): Erlotinib in previously treated non-smallcell lung cancer. New Eng. J. Med., 353, 123-132.

Siegelin, M.D. and Borczuk, A.C. (2014): Epidermal growth factor receptor mutations in lung adenocarcinoma. Lab. Invest., 94, 129-137.

Sui, X., Chen, R., Wang, Z., Huang, Z., Kong, N., Zhang, M., Han, W., Lou, F., Yang, J., Zhang, Q., Wang, X., He, C. and Pan, H. (2013): Autophagy and chemotherapy resistance: a promising therapeutic target for cancer treatment. Cell Death Dis., 4, e838.

Vichai, V. and Kirtikara, K. (2006): Sulforhodamine B colorimetric assay for cytotoxicity screening. Nat. Protoc., 1, 1112-1116.

Wang, J., Deng, X., Zhang, F., Chen, D. and Ding, W. (2014): ZnO nanoparticle-induced oxidative stress triggers apoptosis by activating JNK signaling pathway in cultured primary astrocytes. Nanoscale Res. Lett., 9, 117.

Yang, Z.J., Chee, C.E., Huang, S. and Sinicrope, F.A. (2011): The role of autophagy in cancer: therapeutic implications. Mol. Cancer Ther., 10, 1533-1541.

Yu, K.N., Yoon, T.J., Minai-Tehrani, A., Kim, J.E., Park, S.J., Jeong, M.S., Ha, S.W., Lee, J.K., Kim, J.S. and Cho, M.H. (2013): Zinc oxide nanoparticle induced autophagic cell death and mitochondrial damage via reactive oxygen species generation. Toxicology In Vitro, 27, 1187-1195.

Zhang, Y., Nayak, T.R., Hong, H. and Cai, W. (2013): Biomedical applications of zinc oxide nanomaterials. Curr. Mol. Med., 13, 1633-1645. 